\title{
Understanding Mechanical Properties in Fused Filament Fabrication of Polyether Ether Ketone
}

\author{
Jing $\mathrm{Pu}{ }^{1}$ \\ Centre for Additive Manufacturing, Composites Research Group, University of Nottingham, Nottingham, UK, NG8 1BB \\ Claire McIlroy \\ School of Mathematics 83 Physics, University of Lincoln, Lincoln, UK, LN6 7TS
}

Arthur Jones

Composites Research Group, University of Nottingham, Nottingham, UK, NG8 1BB

Ian Ashcroft

Centre for Additive Manufacturing, University of Nottingham, Nottingham, UK, NG8 1BB

\begin{abstract}
Using dynamic mechanical analysis (DMA), we investigate differences in the mechanical properties of a single-filament wall of polyether ether ketone (PEEK) constructed using fused filament fabrication (FFF) under a range of different printing conditions. Since PEEK is a semi-crystalline polymer, we employ a non-isothermal quiescent crystallization model, informed by infra-red (IR)-imaging measurements, to understand our findings. We propose that, under typical FFF cooling conditions, the weld region between filaments remains amorphous. In contrast, the core of the filament has increased time above the glass transition temperature allowing for a significant crystal fraction to develop. We correlate the predicted crystal fraction to a storage modulus using the Halpin and Kardos model. With only a single model fitting parameter we can make reasonable predictions for the perpendicular and parallel storage moduli measured via DMA over a range of printing conditions. This work provides a foundation for optimising crystallization for the mechanical performance of the FFF printed PEEK.
\end{abstract}

Keywords: polyether ether ketone (PEEK), fused filament fabrication (FFF), quiescent crystallization model, infra-red (IR) thermography, dynamic mechanical analysis (DMA)

\section{Introduction}

Additive Manufacturing (AM), often referred to as 3D printing, is an advanced manufacturing field that has found interesting industrial application in recent years $[1,2,3]$. AM refers to the manufacture of functional parts in a layer-by-layer manner to form a physical part embodying a digital-defined geometry. AM enables near net shape fabrication, reducing the need for material removal operations [4]. Another feature of AM is the ability to realize complex geometry without the added cost of subtractive manufacturing methods, thereby enabling completely new designs and functionalities [5]. Fused filament fabrication (FFF) is one of the most popular AM methods because of its low material and equipment costs $[6]$. FFF is a filamentbased material extrusion technology where a temperaturecontrolled nozzle extrudes thermoplastic material onto a substrate [7].

\footnotetext{
${ }^{1}$ The first and second authors equally contribute to this research.
}

Compared with polymers such as polylactic acid (PLA), polyamide (PA) and acylonitrile butadiene styrene (ABS), polyether ether ketone (PEEK) is a material with a high melting temperature, a low thermal conductivity, and a generally narrow processing window. This makes PEEK difficult to process with FFF printing $[8,9]$. In contrast to other FFF polymers, reports have shown that using FFF to construct PEEK parts may cause excessive thermal stresses, which result in the parts cracking $[9,10]$. Yang et al [11] and $\mathrm{Wu}$ et al [12] compared the mechanical properties of PEEK artefacts made by the FFF printing process with those made by injection moulding. They found a lower mechanical strength for the FFF printed artefacts compared with the injection molded artefacts. Depending on the thickness and angle between deposited filaments, the tensile strength of FFF-printed samples was found on average to be $45 \%$ of injection molded PEEK; the bending strength was $71 \%$ and the compressive strength was $34 \%$ of injection molded PEEK. This proved to be re- 
lated to the processing conditions. Hence, understanding the relationship between the printing parameters and the quality of the manufactured parts is important if the superior polymeric properties of PEEK are to be achieved by FFF. Since PEEK is a semi-crystalline polymer, its mechanical properties depend strongly on the degree of crystallinity $[13,14]$. Studies presented in Refs $[15,16,17]$ show that slower cooling rates can increase the degree of crystallinity and therefore increase the tensile strength and elastic modulus. Liao et al [18] show that due to the repeated heating and cooling cycles that occur during a layered build, the FFF printed layer is always under a unstable crystallization until the printing process is finished. By adjusting the thermal processing parameters [19, 20], it is possible to obtain a wide range of crystallinity fractions in the samples due to the crystallization kinetics of PEEK. Thus, appropriate design of the processing conditions will allow manufacture of PEEK parts with the maximum possible crystal fraction of $50 \%$ [21]. For this reason, in this paper we focus on investigating the crystal growth of PEEK during FFF.

We apply dynamic mechanical analysis (DMA) to measure the mechanical performance of a single-filament PEEK wall printed under various conditions. Moreover, we perform the DMA test both parallel and perpendicular to the print direction to reveal cross-sectional differences in the mechanical properties, i.e. differences between properties in the weld regions compared to within the filaments. In order to understand these disparities, we employ a numerical quiescent crystallization model. As this model requires knowledge of the thermal history, we also conduct infra-red (IR) thermography to analyze how cooling varies with print speed and print temperature [22]. Using classic multi-phase composite solid theory [13], we relate the degree of crystallinity predicted by the model to a storage modulus, and consequently the results of our DMA testing. Thus, we have a method to relate the printing parameters to the mechanical properties of the printed parts, and therefore a foundation for optimizing crystallinization for mechanical performance.

\section{Experimental Methods}

\subsection{Material Properties}

The PEEK filament used in the experiments was the Victrex $450 \mathrm{G}$ with standard melting viscosity, acquired from iMaker Ltd (London, UK). It has a $1.75 \mathrm{~mm}$ diameter with natural colour. PEEK is a semi-crystalline polymer with a glass transition temperature $\left(T_{g}\right)$ of 143 ${ }^{\circ} \mathrm{C}$, and a melting temperature $\left(T_{m}\right)$ of $343{ }^{\circ} \mathrm{C}$. The water absorption of this PEEK at a room temperature of $23{ }^{\circ} \mathrm{C}$ is $0.45 \%$. The PEEK filament was kept in a box with desiccant before printing. The thermal diffusivity of PEEK at $T_{m}$, which is denoted $\alpha$, is $1.27 \times 10^{-7} \mathrm{~m}^{2} \mathrm{~s}^{-1}$ [23]. The storage modulus of fully amorphous PEEK is typically around 0.07 GPa at a plateau [24].

\subsection{FFF Printing}

A CreatBot F430 printer (Henan Suwei Electronic Technology Co., Ltd, Henan, China) was used for the printing. The printer is equipped with a nozzle of diameter size 0.4 $\mathrm{mm}$ that can be heated up to $420{ }^{\circ} \mathrm{C}$. The chamber and printbed of this printer can both be heated to keep the environment at the required temperature. The maximum printbed temperature is $90^{\circ} \mathrm{C}$.

In order to facilitate thermography measurements using infra-red (IR) imaging, as in Ref [22], and enable a more accurate comparison with McIlroy et al's model [25] discussed in Section. 4, we chose to mechanically test a rectangular sample that is a single filament thick. The DMA tension test fixture accommodates samples up to 5 $\times 10 \mathrm{~mm}$. Thus, to create the test sample we designed a print path to print a hollow rectangular box, with walls consisting of a single filament, as shown in Fig. 1(a). The test samples were then cut using a pair of sharp scissors from the walls of the box, as shown in Fig. 1(b). Note that we varied the height of the box in the $z$-direction according to the test direction; a height of $5 \mathrm{~mm}$ (33 layers) was used for the parallel test, compaired with a height of 15 mm (97 layers) required for the perpendicular test. This is shown schematically in Fig. 1(c).

During printing, it was found that only certain printing parameters yielded a printable part; adjacent filaments did not bond well if the print speed was too fast, or if the print temperature was too low. For this reason, we printed samples in a print temperature $\left(T_{N}\right)$ range of $390{ }^{\circ} \mathrm{C}$ to $420{ }^{\circ} \mathrm{C}$ and with print speeds of $3 \mathrm{~mm} / \mathrm{s}, 6 \mathrm{~mm} / \mathrm{s}$ and $18 \mathrm{~mm} / \mathrm{s}$ in the $x y$-plane. Under these conditions, samples generally had good dimensional accuracy and structural integrity, with no visible flaws or thermal distortion. Within this print speed range, the width of a deposited filament is consistently $0.5 \mathrm{~mm}$ with approximately $2 \%$ error. This window for which the road width is independent of print speed has previously been considered in Ref [26]. The machine control data (G-code) was initially generated by the software called CreatWare 6.4.7, which is associated with the CreatBot F430 printer. Subsequently the print path G-code was modified using the Repetier-Host software (Hot-World GmbH \& Co. KG, Willich, Germany). The G-code was modified to enable printing of the single filament walls shown in Fig. 1(a). Note that the print strategy ensures that all layers in a wall are printed in the same direction for consistent cooling profiles in the form of temperature distribution of the $x$-direction. The printer chamber was pre-heated until the printbed temperature became stable at $90{ }^{\circ} \mathrm{C}$, then the printer front door was opened to make the IR-imaging measurements described in the Section. 2.3.

\subsection{IR Thermography}

Since the temperature profile is required as an input for the crystallization model described in Section. 4, we conducted IR thermography across all three print speeds. 


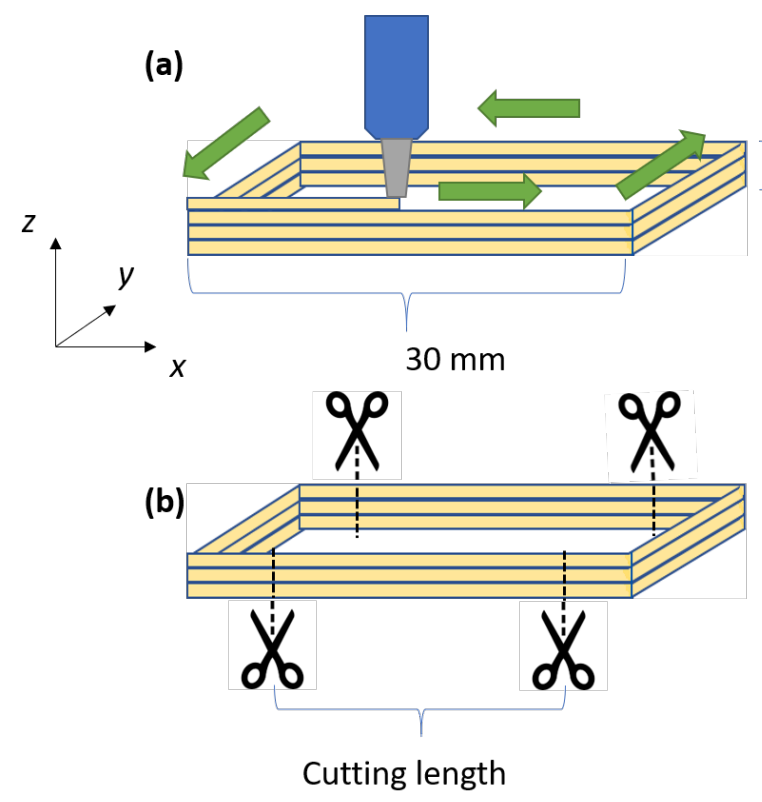

height of the printed box (c)

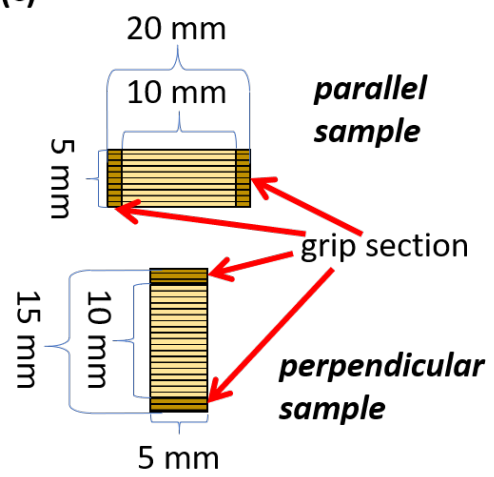

Figure 1: Fabrication of DMA test samples from a printed box with walls that are a single filament thick, with (a) the printing direction, (b) cutting lines, and (c) final sample dimensions.

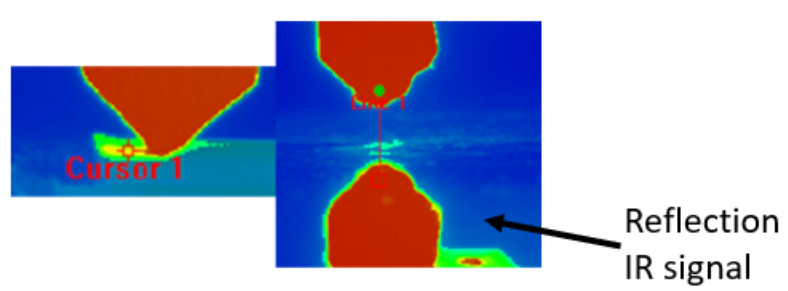

(a)

Figure 2: IR image of printing (a) printing with loaded nozzle (b) printing with unloaded nozzle

With any printable conditions, the thermal history of the filaments could be IR-imaging measured. IR thermography can be used to measure two-dimensional temperature profiles in the FFF process [22]. It gives a surface measurement of the temperature profile. Using the notation of Seppala et al [22], the top filament being actively printed is $L_{i+1}$, and the adjacent sub-layer is $L_{i}$. The mid-point of these adjacent filaments is the weld line $W_{i}$. Due to the curvature of the filaments, it is not feasible to report the IR-imaging measurement at the weld line directly. Thus, the weld temperature was calculated as the average value for two adjacent filaments i.e. $W_{i}=\left(L_{i+1}+L_{i}\right) / 2$.

Temperature profiles were measured with the FLIR SC-7600 IR camera from FLIR Systems (Oregon, U.S.). The frame rate was set to $100 \mathrm{~Hz}$ for the recording. The region of interest (ROI) is three by three pixels and is placed at the middle of each layer during the printing process. The ROI cursor used to locate the first filament $L_{i+1}$

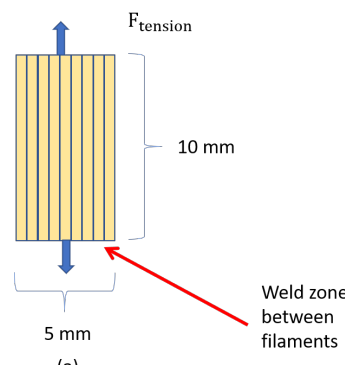

(a) (b)

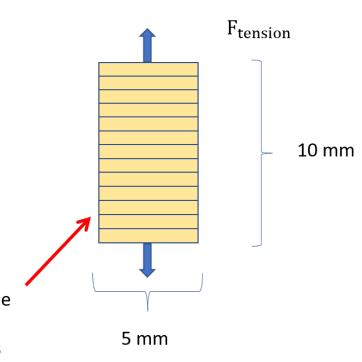

Figure 3: Schematic of DMA test samples. The valid test size is less than $5 \mathrm{~mm}$ width and $10 \mathrm{~mm}$ length (a) test sample with parallel load direction (b) test sample with perpendicular load direction

temperature is shown in Fig. 2(a). The IR signals were converted into temperature directly using standard thermographic measurement techniques that account for the emissivity of the object, the reflected apparent temperature, the distance between the object and the camera, the relative humidity, and the temperature of the atmosphere $[27]$.

Since crystallization ceases at the $T_{g}$, we stopped the IR-imaging measurement once the temperature decreased below $T_{g}$. The reflection IR signals were corrected using the method of Seppala et al [28]. This reflection-correction method requires subtracting the signal obtained from a pass of an unloaded nozzle. By this means we were able to obtain the surface temperature evolution at the filament mid-point under these conditions, as shown in Fig. 2(b).

\subsection{Dynamic Mechanical Analysis}

We used DMA to measure mechanical stiffness. A Dynamic Mechanical Analyzer DMA 8000 from PerkinElmer, 
Inc. (Massachusetts, US) was used. A sinusoidal stress was applied and the strain in the material was measured. The temperature was fixed at room temperature $\left(23^{\circ} \mathrm{C}\right)$, and the frequency at $1 \mathrm{~Hz} .6$ samples were tested for each print condition, and each test lasted for 5 minutes. The test mode executed was the tension test. The measurements were carried out for samples in which the filaments were either perpendicular (sample with 33 layers) to or parallel (sample with 97 layers) to the loading direction in the DMA test, as illustrated in Fig 3.

\section{Experimental Results}

Fig. 4 shows the results of the perpendicular and parallel DMA tests for the printing conditions detailed in Section. 2.2. Error bars represent the standard deviation from the time-averaged mean, averaged over the 6 samples. Fig. 7 indicates the temperature profile at the weld calculated by the IR-imaging measurement under different print conditions. First, from the DMA results, we note that both the perpendicular, $E_{\text {perp }}$, and parallel, $E_{\text {par }}$, storage moduli are greater than the storage modulus of the amorphous material $\left(E_{a}=0.07 \mathrm{GPa}[24]\right)$. We also find that $E_{\text {par }}$ is on average between $24 \%$ to $40 \%$ greater than $E_{\text {perp. }}$. Similar anisotropy has been observed by Capote et al [29] for acrylonitrile butadiene. Here tensile, compression and torsion tests were performed to calculate a failure envelop. Considerable interactions were found between the shear stresses applied in directions parallel and

(a)
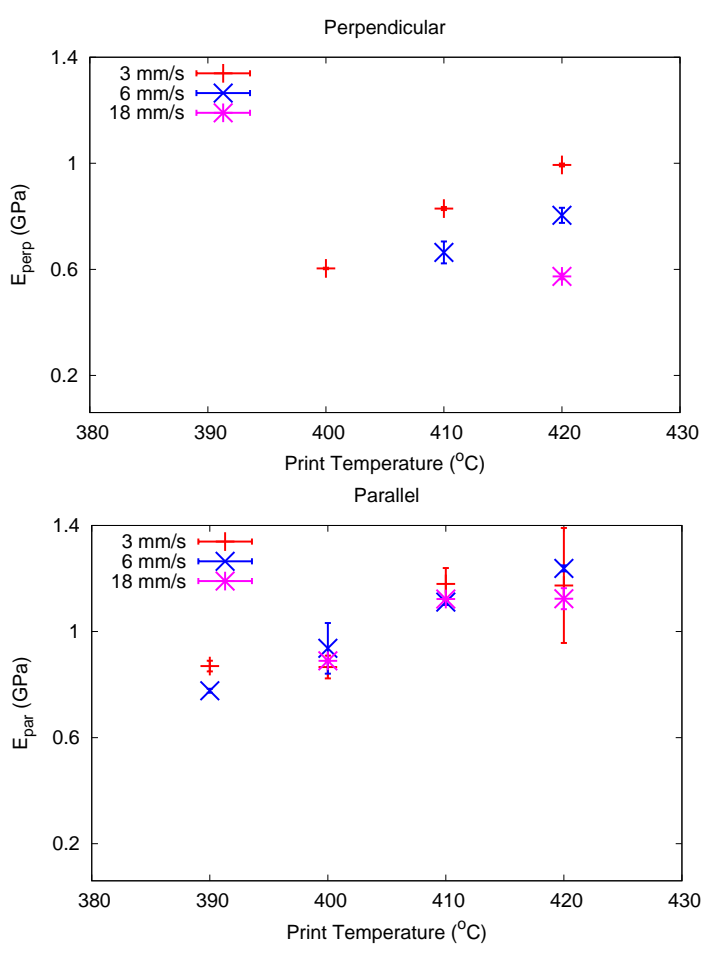

(b)

Figure 4: Storage modulus of PEEK single-filament wall tested via DMA in the (a) perpendicular, $E_{\text {perp }}$, and (b) parallel, $E_{\text {par }}$, directions for the prescribed print conditions. perpendicular to the deposited filaments in a typical dogbone specimen. It can also be seen in Fig. 4 that both $E_{\text {par }}$ and $E_{\text {perp }}$ depend on the print temperature, with higher print temperatures yielding greater storage moduli. Finally, we observe that on average there is little dependence on print speed for $E_{\text {par }}$, whereas $E_{\text {perp }}$ decreases with print speed. The IR-imaging measurement are used to predict the cooling profile at the filament centre in Section. 4.3. In order to understand these results, we propose the following hypothesis.

Since PEEK is a semi-crystalline polymer, it is known that an increase in storage modulus may originate from an increase in crystal fraction $[13,14,30]$. Indeed, both $E_{\text {par }}$ and $E_{\text {perp }}$ are greater than $E_{a}$, and we observe a change in transparency during the printing, which indicates that the filament crystallizes to some degree during cooling. Thus, we propose that the increase in $E_{\text {par }}$ with print temperature seen in Fig. 4(b) is due to an increase in the degree of crystallinity. Furthermore, since $E_{\text {par }}>E_{\text {perp }}$, we propose that the cores of the filaments achieve a greater degree of crystallinity than that of the weld regions during cooling. This reasoning for this assumption will become apparent in Section. 4. Next, we explore this hypothesis via a numerical model that considers non-isothermal, quiescent crystallization under the prescribed printing conditions.

\section{A Numerical Model}

\subsection{Overview}

Here we introduce a numerical model based on the work of McIlroy et al [25]. In line with our hypothesis, we develop a model to predict the crystallization kinetics of a singly-deposited filament of PEEK under typical printing conditions, based on the Schneider rate equations [31]. Since the process is non-isothermal, we account for spatial variations in the temperature evolution via the axisymmetric heat equation, together with a boundary condition informed by the IR-imaging measurements.

In particular, we make predictions of the final crystal fraction at the centre of a deposited filament, $\phi_{f}$, and at the surface of a deposited filament, $\phi_{w}$, which corresponds to a weld region within a fully constructed wall. These predictions of crystallinity are related to the storage moduli in the filament and in the weld, denoted $E_{f}$ and $E_{w}$, respectively, via the composite solid theory of Halpin \& Kardos et al [13] in Section. 4.4.

In order to compare the DMA measurements to the crystallization model we must relate $E_{f}$ and $E_{w}$ to the sample-averaged measurements $E_{\text {par }}$ and $E_{\text {perp }}$. Indeed, by considering the stiffness of heterogeneous structures, we can write

$$
\frac{1}{E_{\text {perp }}}=\frac{1}{z}\left(\frac{z-z_{w}}{E_{f}}+\frac{z_{w}}{E_{w}}\right),
$$

and

$$
E_{\text {par }}=\frac{1}{z}\left(E_{f}\left(z-z_{w}\right)+E_{w} z_{w}\right)
$$


where $z$ is the height of a single filament and $z_{w}$ is the height of a single weld region. See Section. 4.5 for details of this derivation. Note that since the samples are walls with a thickness of a single filament, we have neglected porosity here. Also, Eqs.1 and 2 highlight how variations in $z_{w}$ along the print direction may lead to significant deviations within a sample.

\subsection{Modelling Quiescent Crystallization}

This model considers the homogeneous growth of spherulitic structures via the Schneider rate equations:

$$
\begin{array}{ll}
\dot{\phi_{3}}=8 \pi \dot{N}(T), & \left(\phi_{3}=8 \pi N\right), \\
\dot{\phi}_{2}=G(T) \phi_{3}, & \left(\phi_{2}=8 \pi R_{t o t}\right) \\
\dot{\phi_{1}}=G(T) \phi_{2}, & \left(\phi_{1}=S_{t o t}\right), \\
\dot{\phi_{0}}=G(t) \phi_{1}, \quad\left(\phi_{0}=V_{t o t}\right),
\end{array}
$$

where $N, R_{\text {tot }}, S_{\text {tot }}$ and $V_{\text {tot }}$ are the total number, radii, surface area and volume fraction of spherulites that grow over time. The crystal growth rate, $G$, and nucleation rate, $\dot{N}$, depend on the temperature profile, $T$, as discussed below. To account for space filling, the Avrami equation is employed:

$$
\xi=1-\exp \left(-\phi_{0}\right)
$$

where $\xi$ is the degree of space filling. Here the degree of space filling is related to the crystal fraction via:

$$
\phi=V_{\max } \xi
$$

where $V_{\max }$ is the maximum degree of crystallinity for PEEK $V_{\max } \approx 0.3$ [21]. The Schneider rate equations are solved via Euler's method with a non-isothermal temperature evolution measured via IR-imaging measurement. The temperature dependence of the crystal growth and nucleation rates are informed by experimental measurements found in the literature, as discussed below:

The temperature dependence of the crystal growth rate takes the form [32]:

$$
G(T)=G_{\max } \exp \left(b_{\mathrm{G}}\left(T-T_{\mathrm{G}, \mathrm{ref}}\right)\right)^{2}
$$

where constants $G_{\max }, b_{\mathrm{G}}$ and $T_{\mathrm{G} \text {,ref }}$ are chosen by fitting the growth rate prediction to optical microscopy measurements in Ref. [14]. The crystal growth curve is shown in Fig 5.

The temperature dependence of the nucleation rate takes the classic form [33]:

$$
\dot{N}=\dot{N}_{0} \exp \left(-\frac{n_{a}}{T-T_{g}}\right) \exp \left(-n_{b} \frac{T_{m}^{4}}{\left(T_{m}-T\right)^{2}}\right)
$$

where $\dot{N}_{0}, n_{a}$ and $n_{b}$ are fitting coefficients. To find coefficients $n_{a}$ and $n_{b}$, we employ experimental measurements of the crystallization half time [30], which is assumed to be equivalent to the time to reach $50 \%$ space filling. The half time is measured isothermally using nanocalorimetry

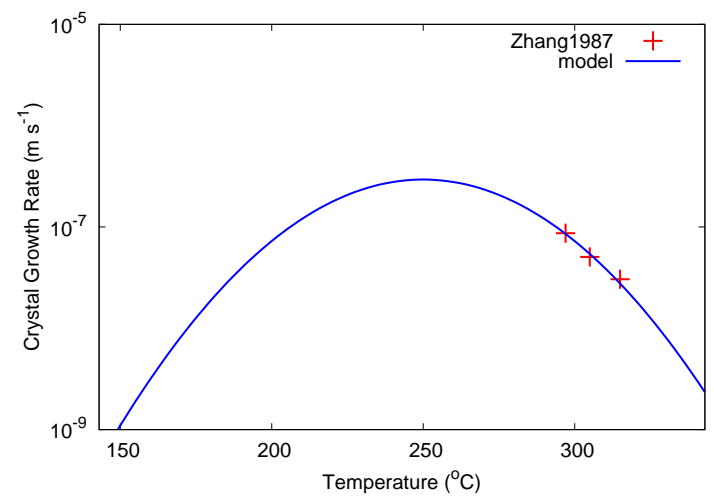

Figure 5: Crystal growth rate given by Eq. (9) with $G_{\max }, b_{G}$ and $T_{\mathrm{G}, \text { ref }}$ fit to Ref. [14]

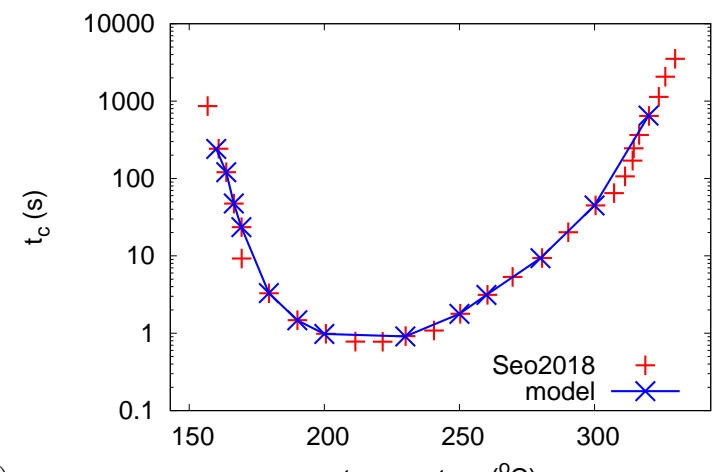

(a)

temperature $\left({ }^{\circ} \mathrm{C}\right)$

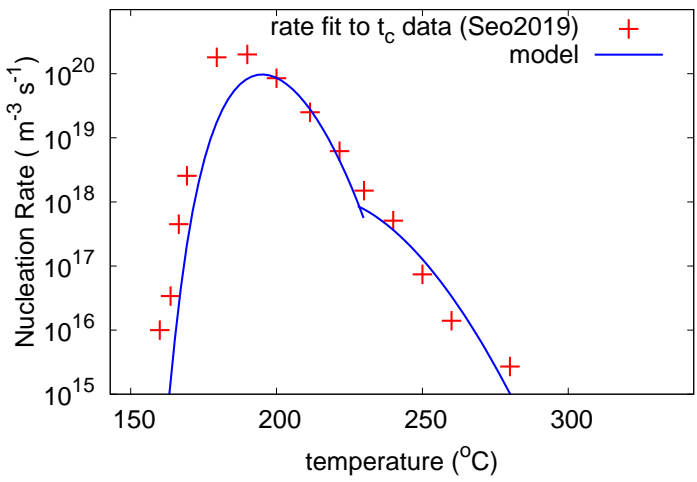

Figure 6: (a) Crystallization half time measured using isothermal nanocalorimetry [30] and model prediction based on solving the Schneider rates equations (Eq.6) and fitting the data for $\dot{N}$. (b) Parameters $\dot{N}_{0}, n_{a}$ and $n_{b}$ are chosen by fitting Eq.10 to the predictions for the nucleation rate.

over a discrete range of temperatures. Thus, by solving the isothermal Schneider rate equations Eq. (6), the nucleation rate $\dot{N}$ can be chosen to fit the experimental data of the half time, as shown in Fig. 6(a). Once $\dot{N}$ is known for a discrete temperature range, parameters $n_{a}$ and $n_{b}$ can be found by fitting Eq. (10), as shown in Fig.6(b).

\subsection{Modelling Temperature Profile}

The IR-imaging technique discussed in Section. 2.3 provides a measurement of the temperature at the filament surface. Whilst this is sufficient to make predictions for 

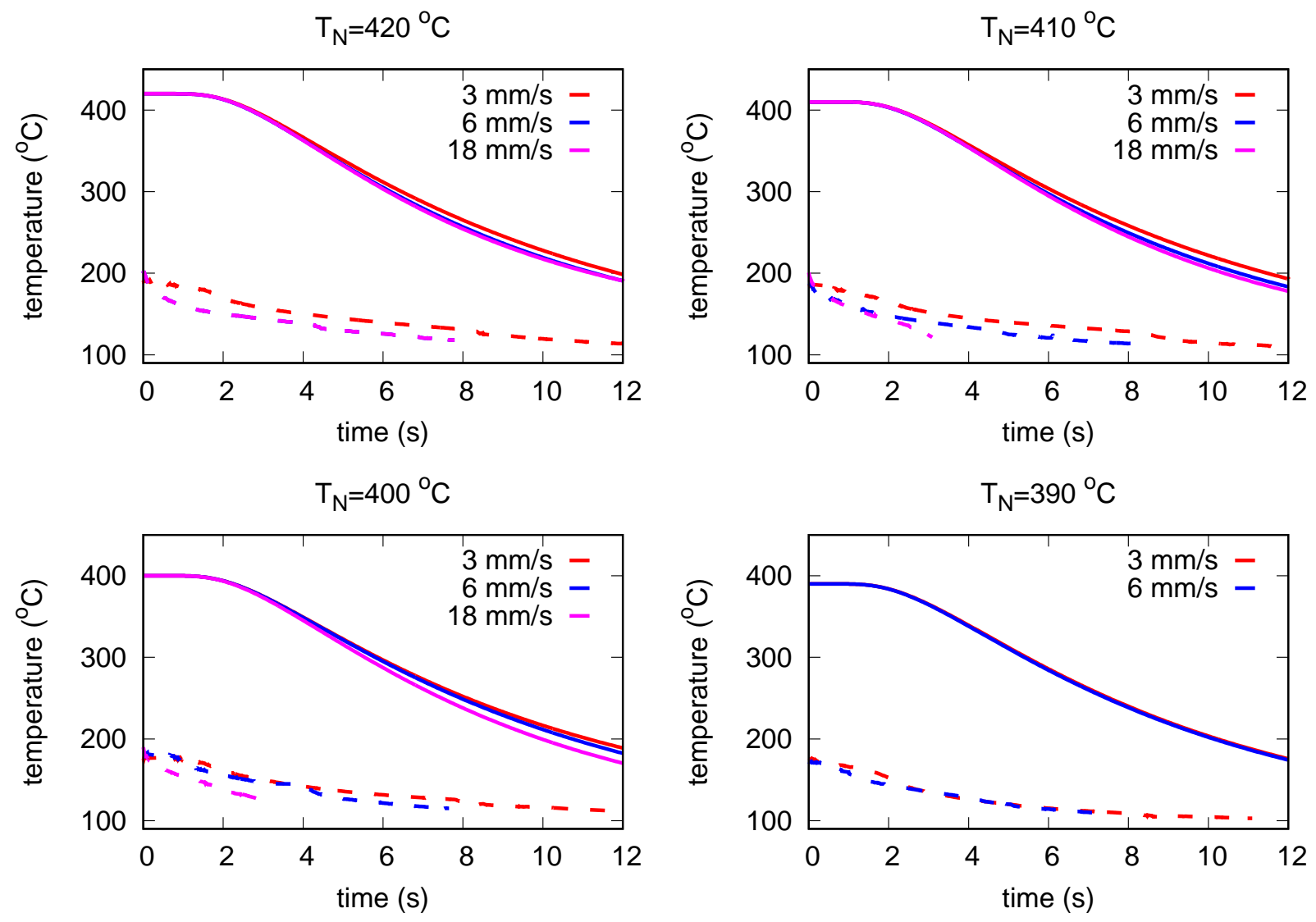

Figure 7: Cooling profile at the filament centre as predicted by solving Eq.(11) (solid line) with a boundary condition specified by the IR-imaging measurement at the weld (dashed line) for each printing condition. The model fitting parameter $b$ is chosen to fit measurements of the modulus, as discussed in Section. 5 .

the crystallization kinetics in the weld region, understanding properties within the filament requires knowledge of the temperature evolution at the filament centre.

To make a prediction of the temperature evolution at the filament centre, we employ the axisymmetric heat equation:

$$
\frac{\partial T}{\partial t}=\alpha \frac{1}{r} \frac{\partial}{\partial r}\left(r \frac{\partial T}{\partial r}\right),
$$

where $\alpha$ is thermal diffusivity. Here, we neglect the effects of latent heat for simplicity.

First, Eq. (11) is non-dimensionalized; the temperature scale is chosen to be the nozzle temperature $T_{N}$, the length scale, $R$, is chosen to be the half-height of a singlydeposited filament. The time scale is governed by the thermal diffusivity time scale:

$$
\tau_{\alpha}=b \frac{R^{2}}{\alpha}
$$

where $b$ is a model fitting parameter. We allow the thermal diffusivity time scale to vary in this way for the following reasons:

1. Thermal diffusivity, $\alpha$, is strongly temperature dependent and may vary in orders of magnitude near to the melting point.
2. The length scale, $R$, may vary due to the ellipticity of the printed filament.

3. The filament may retain heat due to the deposition of multiple filaments.

4. The filament may retain heat due to latent heat effects.

We justify our choice for $b$ in the Section. 5 .

We solve Eq. (11) numerically with initial condition $T(r, t=0)=T_{N}$. The filament is assumed to cool axisymmetrically, the model makes no distinction between filament-filament and filament-air. The boundary condition at the filament surface is given by:

$$
T_{\text {surf }}=T_{0} \exp (-\beta t)+T_{\mathrm{a}}
$$

where $T_{0}, \beta$ and $T_{\mathrm{a}}$ are chosen by fitting the IR-imaging measurement at the weld. In this way we obtain the temperature evolution profile at $r=0$ i.e. at the centre of the filament. This temperature profile can then be employed in the Schneider rate equations (Eq. (6)) to make predictions of the degree of space filling at the filament centre and consequently the properties originating from the filament core (i.e. $E_{f}$ ).

Fig. 7 shows the temperature profiles used in our crystallization model for each of the printing conditions with 
optimum value $b=b_{\text {fit }}$, as discussed in Section. 5. Note that the temperature profile at the weld is provided by the IR-imaging measurement, whereas the temperature at the centre of the filament is obtained from the calculation described above.

\subsection{Halpin and Kardos Theory}

In the theory of Halpin \& Kardos [13], semi-crystalline polymers are assumed to behave as a multi-phase composite solid to develop an equation to link the crystal fraction to the storage modulus of the material. It is shown that the modulus of a semi-crystalline polymer is comparable to that of a randomly-reinforced polymer, where the geometry of the reinforcement is similar in structure to the crystal morphology. Morphology ranges from spherulites consisting of extended fibrils at low volume fractions, to densely packed lamellae at high volume fractions.

Consider a volume fraction of reinforcement fibres, $v_{r}$, with aspect ratio $l / d$, stiffness $S_{r}$, modulus $E_{r}$, and Poisson ratio $\nu_{r}$. The fibres sit within a matrix of stiffness $S_{m}$, modulus $E_{m}$, and Poisson ration $\nu_{m}$. Typically $S_{r} \sim$ $10^{3} S_{m}$ and $E_{r}=10^{3} E_{m}$.

The stiffness of the material in the fibre direction, $S_{11}$, is given by

$$
\frac{S_{11}}{S_{m}}=\frac{1+2(l / d) \eta_{S 1} v_{r}}{1-\eta_{S 1} v_{r}}
$$

where

$$
\eta_{S 1}=\frac{S_{r} / S_{m}-1}{S_{r} / S_{m}+2(l / d)}
$$

Furthermore, the in-plane shear modulus, $E_{12}$, is given by

$$
\frac{E_{12}}{E_{m}}=\frac{1+\eta_{E} v_{r}}{1-\eta v_{r}}
$$

where

$$
\eta_{E}=\frac{E_{r} / E_{m}-1}{E_{r} / E_{m}+1}
$$

The stiffness transverse to the fibre direction is given by

$$
\frac{S_{22}}{S_{m}}=\frac{1+2 \eta_{S 2} v_{r}}{1-\eta_{S 2} v_{r}}
$$

where

$$
\eta_{S 2}=\frac{S_{r} / S_{m}-1}{S_{r} / S_{m}+2}
$$

The Poisson coefficient is given by the rule of mixtures

$$
\nu_{12}=v_{r} \nu_{r}+\nu_{m}\left(1-v_{r}\right)
$$

where

$$
\begin{aligned}
\nu_{r} & =\frac{S_{r}}{2 E_{r}}-1, \\
\nu_{m} & =\frac{S_{m}}{2 E_{m}}-1 .
\end{aligned}
$$

It is shown that the elastic coefficients for a randomlyreinforced solid can be expressed as

$$
\begin{aligned}
\bar{S} & =4 U_{5}\left(U_{1}-U_{5}\right) / U_{1}, \\
\bar{\nu} & =\left(U_{1}-2 U_{5}\right) / U_{1}, \\
\bar{E} & =U_{5}
\end{aligned}
$$

where the invariant terms are given by

$$
\begin{aligned}
U_{1} & =\frac{1}{8}\left(3 Q_{11}+3 Q_{22}+2 Q_{12}+4 Q_{66}\right), \\
U_{5} & =\frac{1}{8}\left(Q_{11}+Q_{22}-2 Q_{12}+4 Q_{66}\right),
\end{aligned}
$$

with

$$
\begin{aligned}
& Q_{11}=S_{11} /\left(1-\nu_{12} \nu_{21}\right) \\
& Q_{22}=S_{22} /\left(1-\nu_{12} \nu_{21}\right) \\
& Q_{12}=\nu_{12} Q_{22}=\nu_{21} Q_{11}, \\
& Q_{66}=E_{12} .
\end{aligned}
$$

\subsection{Stiffness and Modulus in Heterogeneous Structures}

The stiffness of a material, $S$, is related to the modulus via

$$
S=\frac{E A}{L},
$$

where $E$ is the storage modulus, $A=W T$ is the crosssectional area and $L$ is the length of the sample (Fig.8).
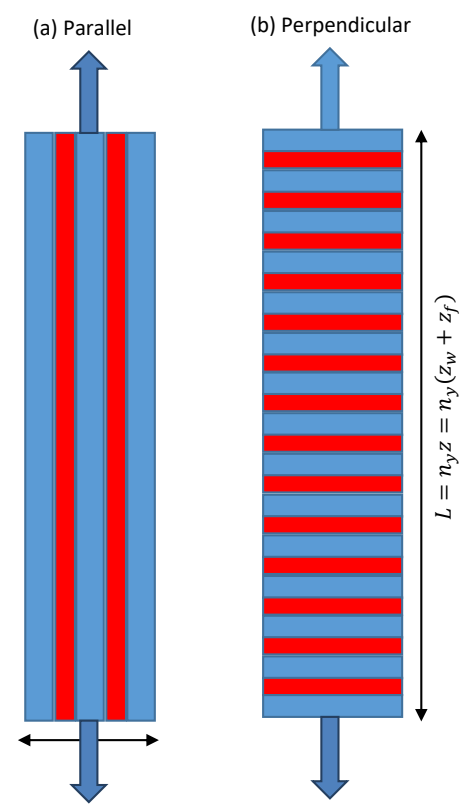

$W=n_{x} z=n_{x}\left(z_{w}+z_{f}\right)$

$T$ is the thickness of the sample

Figure 8: A schematic to show the single-filament wall samples constructed for the (a) parallel and (b) perpendicular DMA tests. Both samples have length $L$, width $W$ and thickness $T$ (equivalent to a single filament). Each individual filament has height $z$; the ratio of amorphous to crystalline material is denoted $z_{w} / z_{f}$. The parallel tests consists of $n_{x}$ filaments, whereas the perpendicular tests consists of $n_{y}$ filaments. 
Our samples are constructed as single-filament walls, with each filament having height $z$. We divide the filament into a core filament region (blue) and a weld region (red) so that $z=z_{f}+z_{w}$. In such hetrogeneous structures, the stiffness is given by

$$
S=S_{f}+S_{w},
$$

where $S_{f}$ and $S_{w}$ denote the stiffness of the filament and weld regions, respectively. Fig. 8 shows how the filaments are constructed for the parallel and perpendicular DMA tests.

For the parallel direction, we have

$$
E_{p a r}=\frac{L}{W T} S_{p a r}=\frac{L}{W T}\left(S_{p a r, f}+S_{p a r, w}\right) .
$$

Since the width of the sample consists of $n_{x}$ filaments, we write

$$
\begin{aligned}
E_{\text {par }} & =\frac{L}{n_{x} z T}\left(\frac{E_{f} n_{x} z_{f} T}{L}+\frac{E_{w} n_{x} z_{w} T}{L}\right), \\
& =\frac{1}{z}\left(E_{f}\left(z-z_{w}\right)+E_{w} z_{w}\right) .
\end{aligned}
$$

Similarly, in the perpendicular direction, we have

$$
\frac{1}{E_{p e r p}}=\frac{W T}{L} \frac{1}{S_{p e r p}}=\frac{W T}{L}\left(\frac{1}{S_{p e r p, f}}+\frac{1}{S_{p e r p, w}}\right) .
$$

Since the length of the sample consists of $n_{y}$ filaments, we write

$$
\begin{aligned}
\frac{1}{E_{\text {perp }}} & =\frac{W T}{n_{y} z}\left(\frac{n_{y} z_{f}}{E_{f} W T}+\frac{n_{y} z_{w}}{E_{w} W T}\right) \\
& =\frac{1}{z}\left(\frac{1-z_{w}}{E_{f}}+\frac{z_{w}}{E_{w}}\right)
\end{aligned}
$$

\section{Discussion}

\subsection{Properties at the Weld}

Fig. 9 shows the model predictions for the final degree of space filling achieved in the weld region under each of the prescribed printing conditions. In particular, we find that, due to fast cooling at the weld, there is insufficient time for crystals to form and the weld remains mostly amorphous i.e. $\xi_{w}<1 \%$. In the case $T_{N}=420^{\circ} \mathrm{C}, 3 \mathrm{~mm} / \mathrm{s}$, the degree of space filling reaches only $2 \%$.

Thus, the model predicts that the mechanical properties at the weld are governed by those of amorphous material. That is, we can assume that the storage modulus at the weld is equivalent to that of the amorphous material, i.e. $E_{w}=E_{a}$. Furthermore, we can define the length scale of the weld region, $z_{w}$, to be the region near to the surface with less than $1 \%$ space filling. This assumption reduces Eqs. 1 and 2 to two unknowns, $E_{f}$ and $z_{w}$, which can thus be written as

$$
E_{f}=\left(E_{a}-E_{p a r}\right)\left(\frac{E_{a}}{E_{p e r p}}-1\right)^{-1}
$$

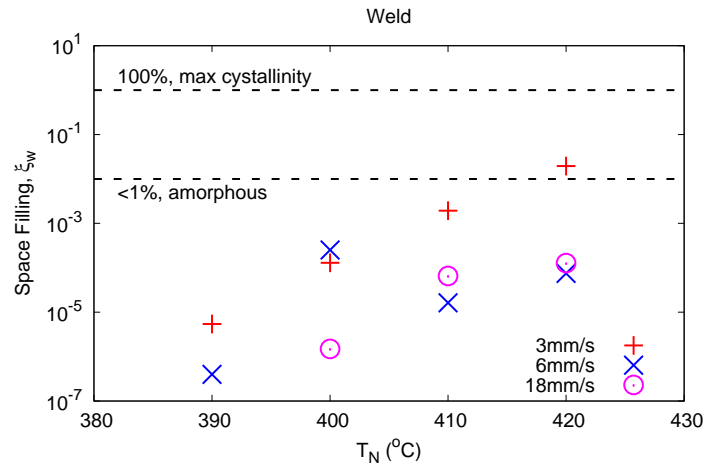

Figure 9: Degree of space filling achieved at the surface of a deposited filament, corresponding to the weld region in a fully constructed wall, as predicted by the model for each of the printing conditions.

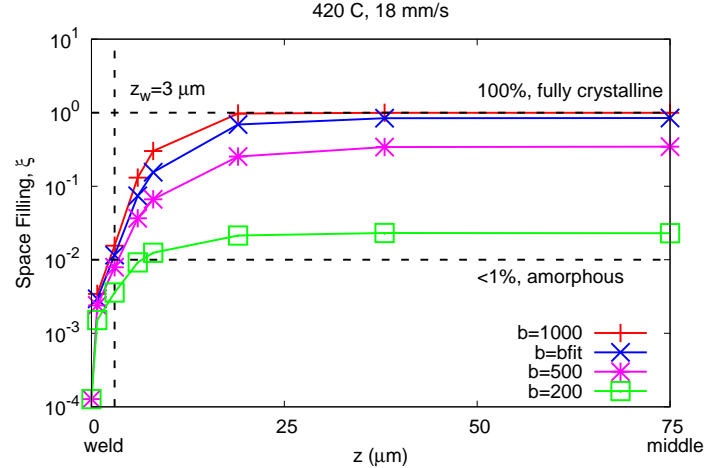

Figure 10: The varying degree of space filling from the centre of the filament to the weld interface predicted by the model for printing condition $420^{\circ} \mathrm{C}, 18 \mathrm{~mm} / \mathrm{s}$ for various values of the fitting parameter b. Eq.41 predicts a length of $z_{w} \sim 3 \mu \mathrm{m}$ for the amorphous weld region.

and

$$
z_{w}=\frac{z\left(E_{p a r}-E_{f}\right)}{E_{a}-E_{f}} .
$$

Hence, using the DMA measurements for $E_{\text {perp }}$ and $E_{\text {par }}$ we can estimate $E_{f}$ and $z_{w}$. In particular, the length scale of the weld region is found to be approximately $z_{w} \sim 1-6$ $\mu \mathrm{m}$, depending on the printing conditions. Consequently, we have an approach for validating our choice for the model fitting parameter, $b$.

In particular, Fig.10 shows how the degree of crystallinity varies spatially from the filament centre to the weld interface for a number of different $b$ values. The print condition is $420{ }^{\circ} \mathrm{C}, 18 \mathrm{~mm} / \mathrm{s}$ and we find similar results across all print conditions. Whilst the weld region remains amorphous $(\xi<1 \%)$ due to rapid cooling at the filament surface, the centre of the filament has sufficient time above $T_{g}$ to develop crystals. We find that the degree of space filling at the centre also depends on the choice of $b$. Thus, $b=b_{f i t}$ is chosen to fit the filament modulus, $E_{f}$, as discussed in Section. 5.2.

\subsection{Properties at the Filament Core}

Fig.11 shows the degree of space filling achieved at the centre of the filament for each of the printing conditions 


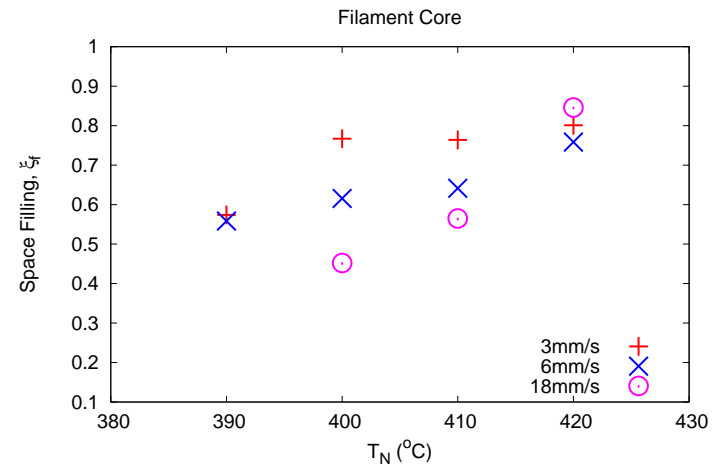

Figure 11: Degree of space filling achieved at the filament centre predicted by the model for each of the printing conditions; $b=b_{\text {fit }}$ is chosen to fit measurements of the filament modulus, $E_{f}$, in Fig. 12.

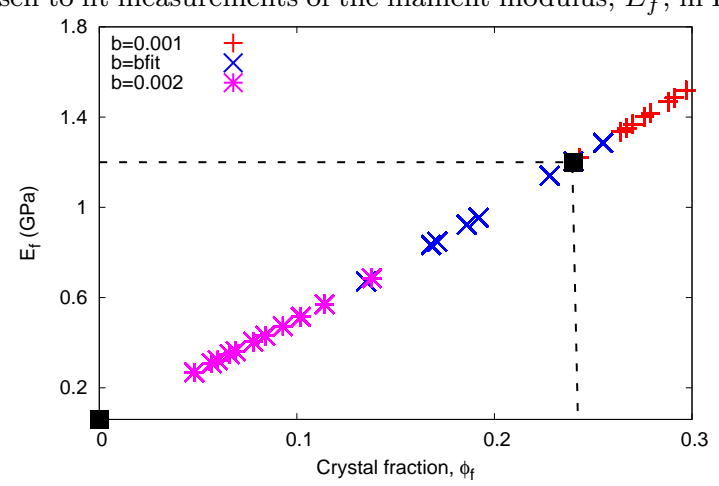

Figure 12: Modulus at the filament centre, $E_{f}$, as predicted by the composite theory of Halpin \& Kardos [13] based on the filament crystal fraction achieved, $\phi_{f}$, under the various printing conditions for different values of $b$. For amorphous material, the crystal fraction is zero and $E_{a}=0.07 \mathrm{GPaPa}$ and from Eq.40, $E_{f}=1.2 \mathrm{GPa}$ for printing condition $420^{\circ} \mathrm{C}, 3 \mathrm{~mm} / \mathrm{s}$, as marked by black squares.

for $b=b_{f i t}$. In general, we find an increase in $\xi_{f}$ with increasing print temperature due to increased time above $T_{g}$. For this fitted value of $b$, the model predicts that the centre of the filament achieves a considerable degree of space filling; in fact, the model predicts $\xi_{f}>40 \%$ for all printing conditions.

Recalling that the degree of space filling is related to the crystal fraction, the increase in storage modulus due to crystal fraction can then be predicted by employing composite theory, as in the work of Halpin \& Kardos [13]. Full details of this model are given in 4.4.

In particular, Fig.12 shows how the filament modulus, $E_{f}$, increases with increasing crystal fraction, $\phi_{f}$. Each data point represents the crystal fraction achieved under the prescribed print conditions and the corresponding modulus for different values of the fitting parameter b. At zero crystal fraction, the modulus corresponds to the amorphous property $E_{a}=0.07 \mathrm{GPa}$, which is marked as the origin in Fig.12. Furthermore, from Eq.40, we find that $E_{f}=1.2 \mathrm{GPa}$ for $420{ }^{\circ} \mathrm{C}, 3 \mathrm{~mm} / \mathrm{s}$. Thus, $b=746$ is chosen so that the model results agree with this value. Thus, Fig.12 highlights cross-sectional disparities in mechanical properties; the modulus at the filament core is

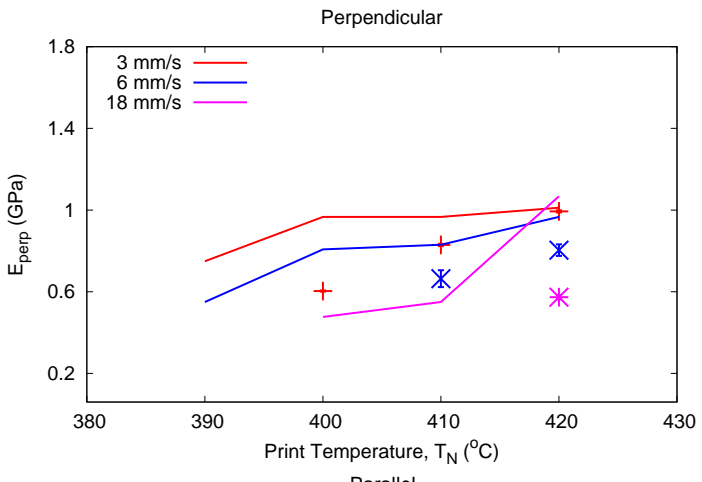

(a)

Parallel

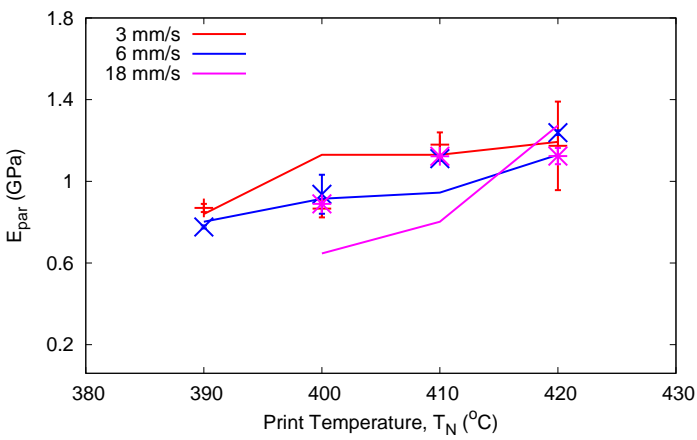

(b)

Figure 13: (a) $E_{\text {perp }}$ and (b) $E_{\text {par }}$ predicted by the model via Eqs.1 and 2 (lines) compared to the DMA measurments (points) for the prescribed print conditions.

two orders of magnitude larger than that at the weld.

\subsection{Comparison to Experimental Measurements}

Now we have determined a suitable value for $b$, we can make direct predictions of $E_{\text {perp }}$ and $E_{\text {par }}$ by assuming $E_{w}=E_{a}$ and substituting the model results for $E_{f}$ and $z_{w}$ into Eqs.1 and 2. Following this, Fig.13 shows that the model predictions using a single $b$ for all print conditions are in reasonable agreement with the DMA measurements. Thus, from only a single parallel and perpendicular DMA measurement, we can make predictions for the perpendicular and parallel storage moduli $E_{\text {perp }}$ and $E_{\text {par }}$ across a range of print conditions, thus enabling us to optimize the print conditions for a required mechanical performance. A better fit could potentially be achieved by fitting $b$ for each print condition, however, this would reduce the practical applicability of the method.

\section{Conclusion}

In this work, we use DMA to investigate mechanical properties in single-filament wall samples of PEEK printed using FFF. We find that the storage modulus increases with print temperature, and that there is some disparity between the perpendicular and parallel measurements. Based on the crystalline nature of PEEK, we hypothesise that these disparities arise due to the differences in the degree of crystallinity achieved under typical FFF conditions, both cross-sectionally and for different printing conditions. 
In particular, we see that PEEK has a narrow processing window under typical FFF conditions, therefore there is little time above the glass transition for crystals to grow.

To investigate our hypothesis, we employ a numerical model of quiescent non-isothermal crystallization, where the temperature history is informed by IR-imaging measurements. The model predicts that the weld regions between printed filaments achieve less than $1 \%$ space filling and are therefore mostly amorphous (Fig. 9). On the other hand, the model predicts that the space filling found in the centre of the filament exceeds $40 \%$ under all printing conditions (Fig. 11) and that higher print temperatures lead to greater degree of space filling. We can relate the filament crystal fraction to a storage modulus by applying the Halpin \& Kardos composite solid model. By employing only a single fitting parameter to one print condition we find reasonable agreement with the DMA measurements across a range of print speeds and temperatures. The model is readily applicable to different materials by selecting appropriate material properties; thermal diffusivity, crystal and nucleation growth rate. The model can also be applied to various print geometries and conditions, provided that a measurement of the temperature evolution can be obtained.

In summary, we have presented a framework for understanding mechanical disparities in FFF printed PEEK. In particular, this crystallization model relates print temperature and print speed to mechanical properties of semicrystalline polymers processed using FFF. Therefore, it provides a foundation for optimizing crystallization for the mechanical performance of FFF-printed PEEK. In the future, we will explore the mechanisms of inter-diffusion and molecular oreintation during FFF printing of PEEK filaments to better understand the amorphous properties in the weld, which will require linear viscoelasticity measurements. We will also seek an independent measurement of crystallinity, for instance via Raman spectroscopy, to compare to the model predictions and investigate the possibility of flow-enhanced crystallization. Furthermore, we will pursue correction of IR-imaging measurement by means of measurement data coming from flash DSC to improve the accuracy in-situ crystallinity measurement. Finally studying the effect of a thermal post-treatment would provide an avenue for achieving higher levels of crystallization in the weld regions.

\section{Acknowledgements}

The first author would like to give thanks to the China Scholarship Council (CSC) for the support of this project. The second author acknowledges funding from the Royal Commission of the Exhibition of 1851 and Royal Society International Exchange Scheme (IESR3/183003). Furthermore, the first author wishes to express her gratitude to Dr. Elizabeth A. Clark and Mr. Christopher I. Strong for sharing their invaluable insights in material science.

\section{References}

[1] K. Wong, A. Hernandez, A review of additive manufacturing. isrn mech eng 2012: 1-10, 2012.

[2] S. H. Huang, P. Liu, A. Mokasdar, L. Hou, Additive manufacturing and its societal impact: a literature review, The International Journal of Advanced Manufacturing Technology 67 (2013) 1191-1203.

[3] W. E. Frazier, Metal additive manufacturing: a review, Journal of Materials Engineering and Performance 23 (2014) 1917-1928.

[4] G. N. Levy, R. Schindel, J.-P. Kruth, Rapid manufacturing and rapid tooling with layer manufacturing $(\mathrm{lm})$ technologies, state of the art and future perspectives, CIRP annals 52 (2003) 589-609.

[5] J. Edgar, S. Tint, Additive manufacturing technologies: 3d printing, rapid prototyping, and direct digital manufacturing, Johnson Matthey Technology Review 59 (2015) 193-198.

[6] A. B. Varotsis, Selecting the right 3D printing process, 3D Hubs, 2019. URL: https://www.3dhubs.com/knowledge-base/selecting-right-3d-printin

[7] S. Hertle, M. Drexler, D. Drummer, Additive manufacturing of poly (propylene) by means of melt extrusion, Macromolecular Materials and Engineering 301 (2016) 1482-1493.

[8] A. Stepashkin, D. Chukov, F. Senatov, A. Salimon, A. Korsunsky, S. Kaloshkin, 3d-printed peek-carbon fiber (cf) composites: Structure and thermal properties, Composites Science and Technology 164 (2018) 319-326.

[9] M. Punchak, L. K. Chung, C. Lagman, T. T. Bui, J. Lazareff, K. Rezzadeh, R. Jarrahy, I. Yang, Outcomes following polyetheretherketone (peek) cranioplasty: systematic review and meta-analysis, Journal of Clinical Neuroscience 41 (2017) 30-35.

[10] X. Deng, Z. Zeng, B. Peng, S. Yan, W. Ke, Mechanical properties optimization of poly-ether-ether-ketone via fused deposition modeling, Materials 11 (2018) 216.

[11] C. Yang, X. Tian, D. Li, Y. Cao, F. Zhao, C. Shi, Influence of thermal processing conditions in $3 \mathrm{~d}$ printing on the crystallinity and mechanical properties of peek material, Journal of Materials Processing Technology 248 (2017) 1-7.

[12] W. Wu, P. Geng, G. Li, D. Zhao, H. Zhang, J. Zhao, Influence of layer thickness and raster angle on the mechanical properties of 3d-printed peek and a comparative mechanical study between peek and abs, Materials 8 (2015) 5834-5846.

[13] J. Halpin, J. Kardos, Moduli of crystalline polymers employing composite theory, Journal of Applied Physics 43 (1972) 22352241 .

[14] Z. Zhang, H. Zeng, Nucleation and crystal growth of peek on carbon fiber, Journal of applied polymer science 48 (1993) 19871995.

[15] M. F. Talbott, G. S. Springer, L. A. Berglund, The effects of crystallinity on the mechanical properties of peek polymer and graphite fiber reinforced peek, Journal of Composite Materials 21 (1987) 1056-1081.

[16] W. I. Lee, M. F. Talbott, G. S. Springer, L. A. Berglund, Effects of cooling rate on the crystallinity and mechanical properties of thermoplastic composites, Journal of Reinforced Plastics and Composites 6 (1987) 2-12.

[17] P. Cebe, S. Y. Chung, S.-D. Hong, Effect of thermal history on mechanical properties of polyetheretherketone below the glass transition temperature, Journal of applied polymer science 33 (1987) 487-503.

[18] C.-Y. Liao, P.-L. Wu, C.-Y. Lee, Customized peek implants with microporous and surface modification using $3 \mathrm{~d}$ printing, in: International Design Engineering Technical Conferences and Computers and Information in Engineering Conference, volume 59223, American Society of Mechanical Engineers, 2019, p. V004T08A013.

[19] D. J. Blundell, B. Osborn, The morphology of poly (aryl-etherether-ketone), Polymer 24 (1983) 953-958.

[20] C. N. Velisaris, J. C. Seferis, Crystallization kinetics of polyetheretherketone (peek) matrices, Polymer Engineering \& Science 26 (1986) 1574-1581. 
[21] R. Chivers, D. Moore, The effect of molecular weight and crystallinity on the mechanical properties of injection moulded poly (aryl-ether-ether-ketone) resin, Polymer 35 (1994) 110-116.

[22] J. E. Seppala, S. H. Han, K. E. Hillgartner, C. S. Davis, K. B. Migler, Weld formation during material extrusion additive manufacturing, Soft Matter 13 (2017) 6761-6769.

[23] Linseiis, Determination of https://www.linseis. com/en/properties/thermal-conductivity/.

[24] L. Martineau, F. Chabert, G. Bernhart, T. Djilali, Mechanical behavior of amorphous peek in the rubbery state, 2016 .

[25] C. McIlroy, R. Graham, Modelling flow-enhanced crystallisation during fused filament fabrication of semi-crystalline polymer melts, Additive Manufacturing 24 (2018) 323-340.

[26] P. Geng, J. Zhao, W. Wu, W. Ye, Y. Wang, S. Wang, S. Zhang, Effects of extrusion speed and printing speed on the $3 \mathrm{~d}$ printing stability of extruded peek filament, Journal of Manufacturing Processes 37 (2019) 266-273.

[27] F. Systems, FLIR VideoReport User's manual, 2009. URL: https://www.flir.com.

[28] J. E. Seppala, K. D. Migler, Infrared thermography of welding zones produced by polymer extrusion additive manufacturing, Additive Manufacturing 12 (2016) 71-76.

[29] G. A. M. Capote, N. M. Rudolph, P. V. Osswald, T. A. Osswald, Failure surface development for abs fused filament fabrication parts, Additive Manufacturing 28 (2019) 169-175.

[30] J. Seo, A. M. Gohn, O. Dubin, H. Takahashi, H. Hasegawa, R. Sato, A. M. Rhoades, R. P. Schaake, R. H. Colby, Isothermal crystallization of poly (ether ether ketone) with different molecular weights over a wide temperature range, Polymer Crystallization 2 (2019) e10055.

[31] W. Schneider, A. Köppl, J. Berger, Non-isothermal crystallization crystallization of polymers: System of rate equations, International Polymer Processing 2 (1988) 151-154.

[32] G. W. Peters, L. Balzano, R. J. Steenbakkers, Flow-induced crystallization, Handbook of polymer crystallization (2013) 399-431.

[33] C. R. Snyder, H. Marand, Effect of chain transport in the secondary surface nucleation based flux theory and in the lauritzenhoffman crystal growth rate formalism, Macromolecules 30 (1997) 2759-2766. 